\title{
High temperature superconducting with two doping atoms in La-doped Bi-2201 and Y-doped Bi-2212
}

\author{
H.P. Roeser ${ }^{a}$, , F.M. Huber ${ }^{\text {b }}$, M.F. von Schoenermark ${ }^{\mathrm{a}}$, A.S. Nikoghosyan ${ }^{\mathrm{c}}$ \\ ${ }^{\mathrm{a}}$ Institute of Space Systems, Universitaet Stuttgart, Pfaffenwaldring 31, 70569 Stuttgart, Germany \\ b Steinbeis-Transferzentrum Raumfahrt, Roetestr. 15, 71126 Gaeufelden, Germany \\ ${ }^{\mathrm{c}}$ Department of Microwave and Telecommunication, Yerevan State University, Alex Manoogian 1, Yerevan 375025, Armenia
}

Received 1 November 2008; received in revised form 7 February 2009; accepted 9 February 2009

Available online 19 March 2009

\begin{abstract}
These crystals have two doping patterns given by oxygen excess and doping with an additional atom with projected positions in the $\mathrm{CuO}_{2}$ plane. Both doping elements are necessary for the occurrence of superconductivity. Where the two doping patterns overlap, the point matched locations act as the resonating superconducting pathway and might explain a correlation between $T_{c}$ and the doping structure.
\end{abstract}

(C) 2009 Elsevier Ltd. All rights reserved.

Keywords: High temperature superconductors; Superconducting unit area; Superconducting current channels

\section{Introduction}

In cuprates, the $\mathrm{CuO}_{2}$ plane is a highly symmetrical and a very well ordered system. Bonding lengths and planarity are very well known by X-ray and neutron diffraction experiments. The superconducting process requires the existence of $\mathrm{Cu}^{3+}$-ions in these planes as well as homogeneously distributed oxygen deficiency or excess positions in or near the $\mathrm{CuO}_{2}$ planes. In previous papers [1-4] a strong correlation has been found between the doping distances $(x)$ and the critical transition temperatures $T_{c}$. However, there exist many high temperature superconductors (HTSC) that have an extra doping atom, in addition to the oxygen excess atom.

\footnotetext{
* Corresponding author. Tel.: +4971168562375; fax: +4971168563596 .

E-mail address: roeser@irs.uni-stuttgart.de (H.P. Roeser).
}

In this paper we will analyse if double doped cuprates also follow the correlation Eq. (1) between the doping structure value $(2 x)^{2}$ and $T_{c}$. We have chosen the cuprates La-doped Bi-2201 and Y-doped Bi-2212 which are HTSCs with oxygen excess atoms [2]:

$(2 x)^{2} \cdot n^{-2 / 3} \cdot 2 M_{e f f} \cdot \pi k T_{c}=h^{2}$

\section{Geometry in the superconducting $\mathrm{CuO}_{2}$ plane with two doping atoms}

For double doped HTSCs, the concept of the unit area for one doping element [1-4] needs to be extended for two different doping elements. The oxygen excess contribution is given by $\delta$, its density by $\left(\Sigma_{1}\right)^{-1}$, the number of unit cells per doping atom within the $\mathrm{CuO}_{2}$ plane by $\Sigma_{1}$ and the density distance by $x_{1}$; the extra atom contribution is $\Delta,\left(\Sigma_{2}\right)^{-1}$ and $x_{2}$, respectively, and the resulting double doping, which is a match between 


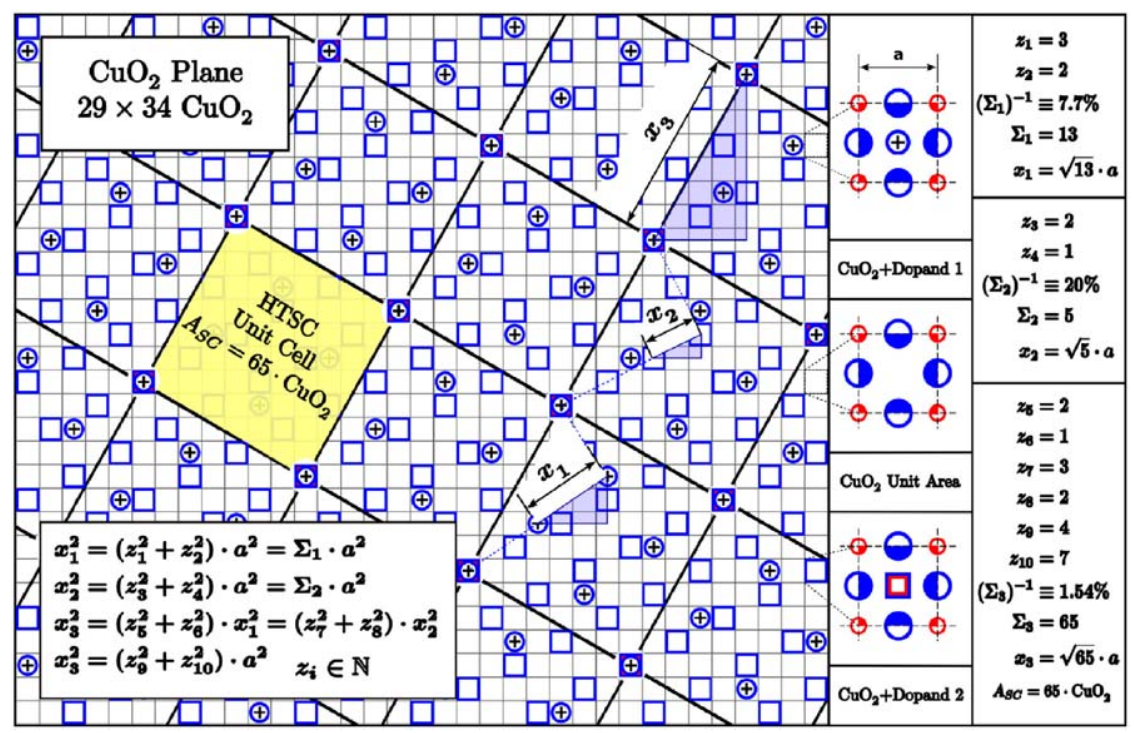

Fig. 1. Illustration of a double doped cuprate where the positions of $\mathrm{CuO}_{2}$ unit areas representing a unit cell which contains two doping atoms are given by a point matching for the two doping patterns which is similar to a Moiré effect.

the oxygen excess positions and the extra atom positions, is given by $\left(\Sigma_{3}\right)^{-1}$ and $x_{3}$.

Cuprates have a tetragonal unit cell structure and the $\mathrm{CuO}_{2}$ unit area has a symmetrical $\mathrm{Cu}$ - and $\mathrm{O}$-atom distribution so that all directions for the superconducting carrier flow should be permitted. As an example, Fig. 1 illustrates the concept for an arbitrary section of the $\mathrm{CuO}_{2}$ plane with $[29 \cdot 34] \mathrm{CuO}_{2}$ unit areas filled with two different homogeneously distributed dopants. The distances $\left(x_{i}\right)$ are given by the hypotenuse of right triangles with $x_{i}=\sqrt{ } \Sigma_{k} \cdot a$ and $\Sigma_{k}=z_{i}{ }^{2}+z_{j}{ }^{2}$ with $z_{i, j} \in$ $\mathbb{N}$, because of the highly periodic and square pattern of the $\mathrm{CuO}_{2}$ plane. The positions of $\mathrm{CuO}_{2}$ unit areas containing two doping atoms are given by a point matching for the two doping patterns which is similar to a Moiré effect leading to

\section{3. $\mathrm{Bi}_{2} \mathrm{Sr}_{1.6} \mathrm{La}_{0.4} \mathrm{CuO}_{6+\delta}(\mathrm{Bi}-2201-\mathrm{La})$}

The parent compound $\mathrm{Bi}_{2} \mathrm{Sr}_{2} \mathrm{CuO}_{6}(\mathrm{Bi}-2201)$, shown in Fig. 2, is not superconducting. When an extra $\mathrm{O}$ atom is added, the overdoped material $\mathrm{Bi}_{2} \mathrm{Sr}_{2} \mathrm{CuO}_{6+\delta}$ is not superconducting either, because of the missing $\mathrm{Cu}^{3+}$-ions in the $\mathrm{CuO}_{2}$ plane [2]. $\mathrm{Cu}^{3+}$-ions are created using $\mathrm{Bi}-2201$ with oxygen excess and replacing $\mathrm{Sr}^{2+}$-ions by trivalent ions like $\mathrm{La}^{3+}, \mathrm{Pr}^{3+}, \mathrm{Nd}^{3+}$, $\mathrm{Sm}^{3+}, \mathrm{Eu}^{3+}, \mathrm{Gd}^{3+}$ at a specific doping level [5]. In this way, the electronic arrangement in the unit cell, containing an excess $\mathrm{O}$-atom and an La-atom, should read $2 \mathrm{Bi}^{3+}+1 \mathrm{La}^{3+}+1 \mathrm{Sr}^{2+}+1 \mathrm{Cu}^{3+}+7 \mathrm{O}^{2-}$ with a chemical formula $\mathrm{Bi}_{2} \mathrm{Sr}_{1-\Delta} \mathrm{La}_{\Delta} \mathrm{CuO}_{6+\delta}$. It has been demonstrated that at an optimum La-doping value of $\Delta=0.4$ a

$$
\begin{array}{ccccccc}
\left(z_{1}^{2}+z_{2}^{2}\right) & \left(z_{5}^{2}+z_{6}^{2}\right) & = & \left(z_{3}^{2}+z_{4}^{2}\right) & \left(z_{7}^{2}+z_{8}^{2}\right) & = & \left(z_{9}^{2}+z_{10}^{2}\right) \\
\left(4^{2}+7^{2}\right) & \left(1^{2}+0^{2}\right) & = & \left(2^{2}+1^{2}\right) & \left(3^{2}+2^{2}\right) & = & \left(4^{2}+7^{2}\right) \\
65 & 1 & = & 5 & 13 & = & 65
\end{array}
$$

The values for $z_{1}$ through $z_{4}$ will be given by the experimentally derived doping values $\delta$ and $\Delta$ or $\Sigma_{1}$ and $\Sigma_{2}$, respectively. For the numbers $z_{5}$ through $z_{10}$ we are looking for the smallest number because the shortest distance is responsible for the highest $T_{c}$ according to Eq. (1). Eq. (2a) represents the numerical values for Bi2201-La. For the known HTSCs, the doping level values $\delta$ and $\Delta$ are larger than $\left(\Sigma_{k}\right)^{-1} \equiv 0.5 \%$ leading to values of $\Sigma_{k}=z_{i}^{2}+z_{j}^{2} \leqslant 200$ for $k=1,2$. transition temperature of $T_{c}=35.5 \pm 2.5 \mathrm{~K}$ can be measured [5-7]. For Bi-2201 it is difficult to obtain high quality single crystals, which explains the large variation of experimental $T_{c}$ values. According to equations in Fig. 1 , the value $\Delta=0.4$ leads to $\left(\Sigma_{2}\right)^{-1}=(1-1.6 / 2.0) \equiv 20 \%$ and $\Sigma_{2}=5=$ $2^{2}+1^{2}=z_{3}^{2}+z_{4}^{2}$ with a doping distance of $x_{2}=\sqrt{ } 5$. $a=1.21 \mathrm{~nm}$ (Table 1$)$. 


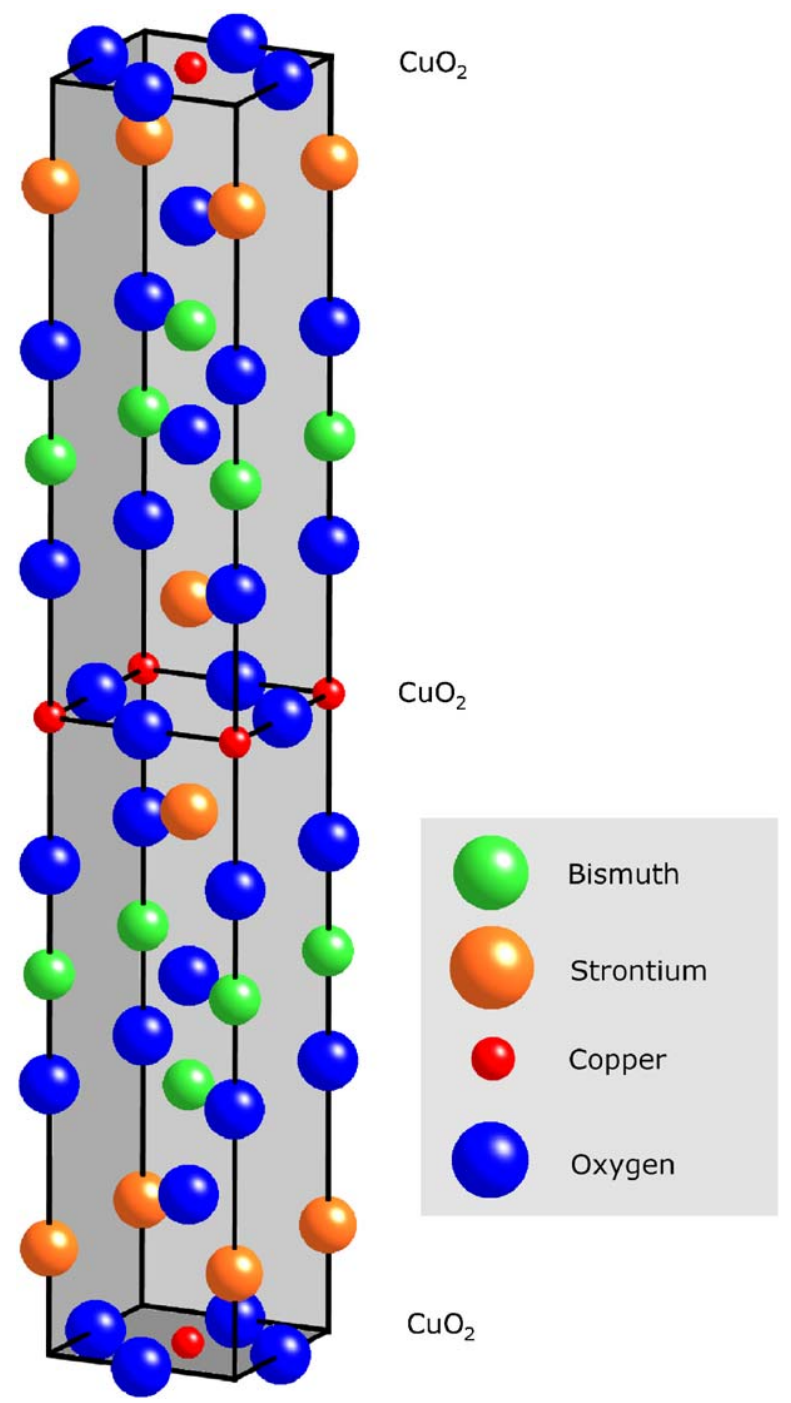

Fig. 2. The unit cell of $\mathrm{Bi}-2201$ consists of $2 \cdot\left[\mathrm{Bi}_{2} \mathrm{Sr}_{2} \mathrm{CuO}_{6}\right]$ and has two $\mathrm{CuO}_{2}$ planes. Note the alternate displacement of the stacking sequence of $\mathrm{CuO}_{2}$ planes.

Superconductivity in the Bi-family requires a very low oxygen excess which is achieved with an appropriate annealing process. The preparation for $\mathrm{Bi}-2201$ is done at temperatures between 650 and $800^{\circ} \mathrm{C}$ within a low oxygen atmosphere and over a long time [5,8,9]. It has been shown that the composition of $\mathrm{Bi}-2201$ follows the nominal compositions in Bi-2212 [5].

Superconductivity is achieved with a value of $\delta \approx 0.1$ with an oxygen content of about $\left(\Sigma_{1}\right)^{-1}=(1-6.0 / 6.1)$ $\equiv 1.6 \pm 0.25 \%$ [8]. The condition given by Eq. (2) that $\Sigma_{1}=\left(z_{1}^{2}+z_{2}^{2}\right)$ permits only certain numbers for $\Sigma_{1}(58,61,64, \mathbf{6 5}, 68,72,73,74)$, but since

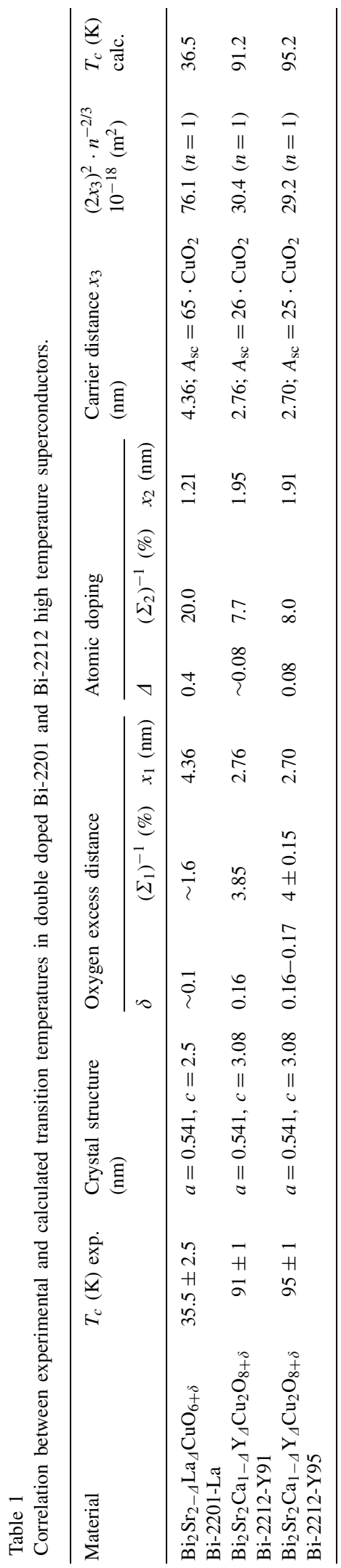




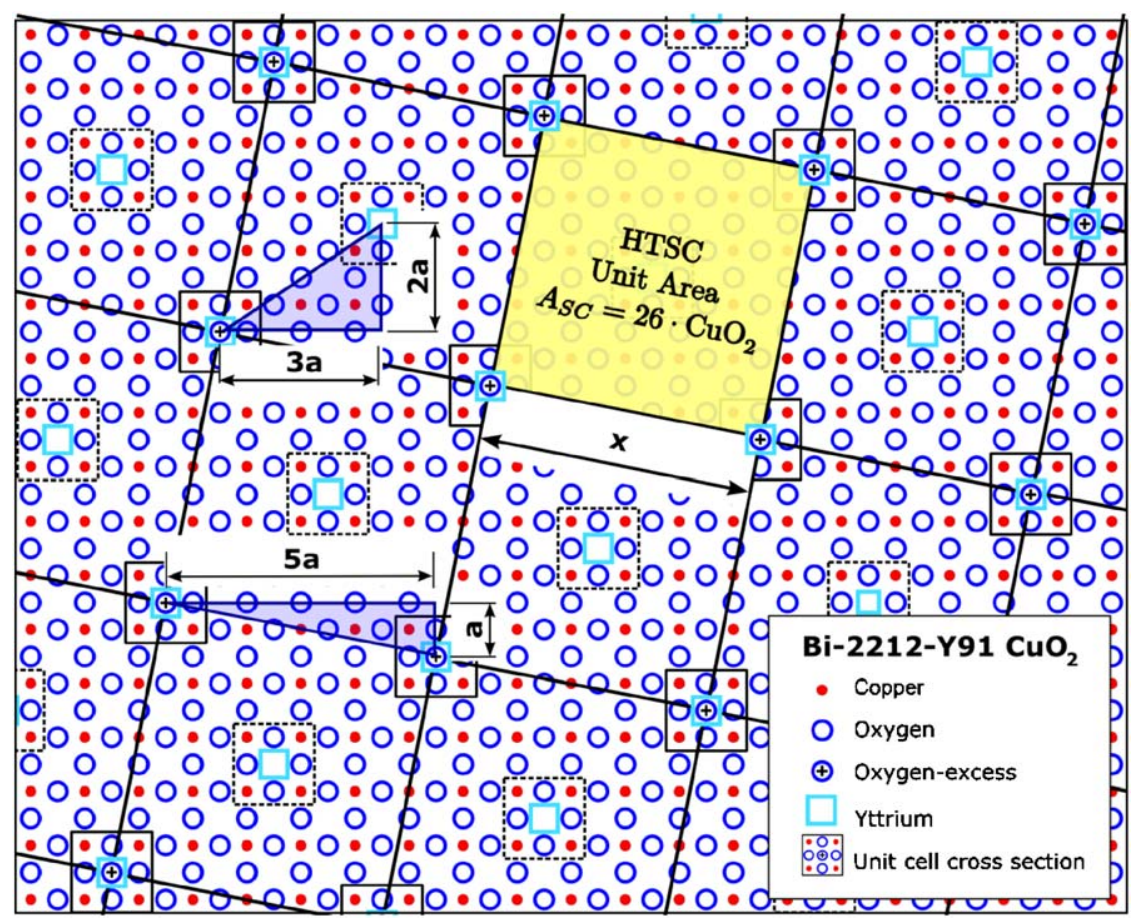

Fig. 3. Superconducting $\mathrm{CuO}_{2}$ plane of $\mathrm{Bi}-2212-\mathrm{Y} 91(a \approx b=0.541 \mathrm{~nm})$. The distance between oxygen excess atoms is the superconducting resonance length $x=2.76 \mathrm{~nm}$. The superconducting unit area has the size of $26 \cdot \mathrm{CuO}_{2}$ unit areas.

$\left(z_{1}^{2}+z_{2}^{2}\right) /\left(z_{3}^{2}+z_{4}^{2}\right)=\left(z_{1}^{2}+z_{2}^{2}\right) / 5=z_{i}$, then $\Sigma_{1}=65$ is the only solution, which is equivalent to $\left(\Sigma_{1}\right)^{-1} \equiv 1.54 \%$. This calculation can be solved with a simple computer program using the conditions of Eq. (2) and leads to the solution of a rectangular pattern with $x_{1}=x_{3}=\sqrt{ } 65 \cdot a=4.36 \mathrm{~nm}$ (Table 1$)$ and a HTSC unit area of $A_{\mathrm{SC}}=65 \cdot \mathrm{CuO}_{2}$ (Fig. 1).

\section{4. $\mathrm{Bi}_{2} \mathrm{Sr}_{2} \mathrm{Ca}_{1-\Delta} \mathrm{Y}_{\Delta} \mathrm{Cu}_{2} \mathrm{O}_{8+\delta}(\mathrm{Bi}-2212-\mathrm{Y})$}

The electronic arrangement for pure $\mathrm{Bi}_{2} \mathrm{Sr}_{2} \mathrm{CaCu}_{2} \mathrm{O}_{8+\delta}$ (Bi-2212) in the unit cell with an excess oxygen atom is $2 \mathrm{Bi}^{3+}+1 \mathrm{Ca}^{2+}+2 \mathrm{Sr}^{2+}+2 \mathrm{Cu}^{3+}+9 \mathrm{O}^{2-}$. This compound forms two superconducting $\mathrm{CuO}_{2}$ planes in which the hole doped cuprate possesses an effective mass of $M_{e f f}=2 m_{e}$. The highest transition temperature for the pure $\mathrm{Bi}-2212$ published so far is $T_{c}=82.5 \mathrm{~K} \pm 0.5 \mathrm{~K}$ $[5,10]$ and has an oxygen excess of $\delta=0.18$ [2]. Reports have been published demonstrating a maximum transition temperature in the range of $T_{c}=90-96 \mathrm{~K}$ for so-called "optimized doping" levels with a value of about $\delta=0.16$. In those cases, Bi-2212 has been additionally doped with a small amount of yttrium or other atoms to yield a well-ordered system [5]. In analysing the published results, there are two different critical transition temperature levels, one at $T_{c}=91 \pm 1 \mathrm{~K}$ for a Y-doping level of about $\Delta=0.08$ resulting in $\mathrm{Bi}_{2} \mathrm{Ca}_{0.92} \mathrm{Sr}_{2} \mathrm{Y}_{0.08} \mathrm{Cu}_{2} \mathrm{O}_{8+\delta}$ [10-13]. The second transition is at $T_{c}=95 \pm 1 \mathrm{~K}$ for the same amount of yttrium but somewhat higher oxygen excess doping level $[5,14,15]$.

\subsection{Bi-2212-Y91}

For the Y-doped case, one chemical formula has two $\mathrm{CuO}_{2}$ planes plus two $\mathrm{SrO}$ and two $\mathrm{BiO}$ planes and a Y-atom in the middle of the unit cell between the two $\mathrm{CuO}_{2}$ planes. Detailed measurements have been done to optimize the oxygen excess doping, resulting in $\delta=0.16$ [16] for $T_{c}=91 \mathrm{~K}$. If the oxygen excess atom is located only between the two $\mathrm{CuO}_{2}$ planes then the relevant density is $\mathrm{O}_{4.16}$ instead of $\mathrm{O}_{8.16}$ leading to $\left(\Sigma_{1}\right)^{-1}=(1-4.00 / 4.16) \equiv 3.85 \%$, so that $\Sigma_{1}=26=5^{2}+1^{2}=z_{1}^{2}+z_{2}^{2}$. The doping distance is then $x_{1}=\sqrt{ } 26 \cdot a=2.76 \mathrm{~nm}$. The electronic arrangement at the position, where we find an oxygen excess atom and a $\mathrm{Y}$-atom at the same time, is given by $2 \mathrm{Bi}^{3+}+\mathrm{Y}^{3+}+2 \mathrm{Sr}^{2+}+\mathrm{Cu}^{2+}+\mathrm{Cu}^{3+}+9 \mathrm{O}^{2-}$. This 


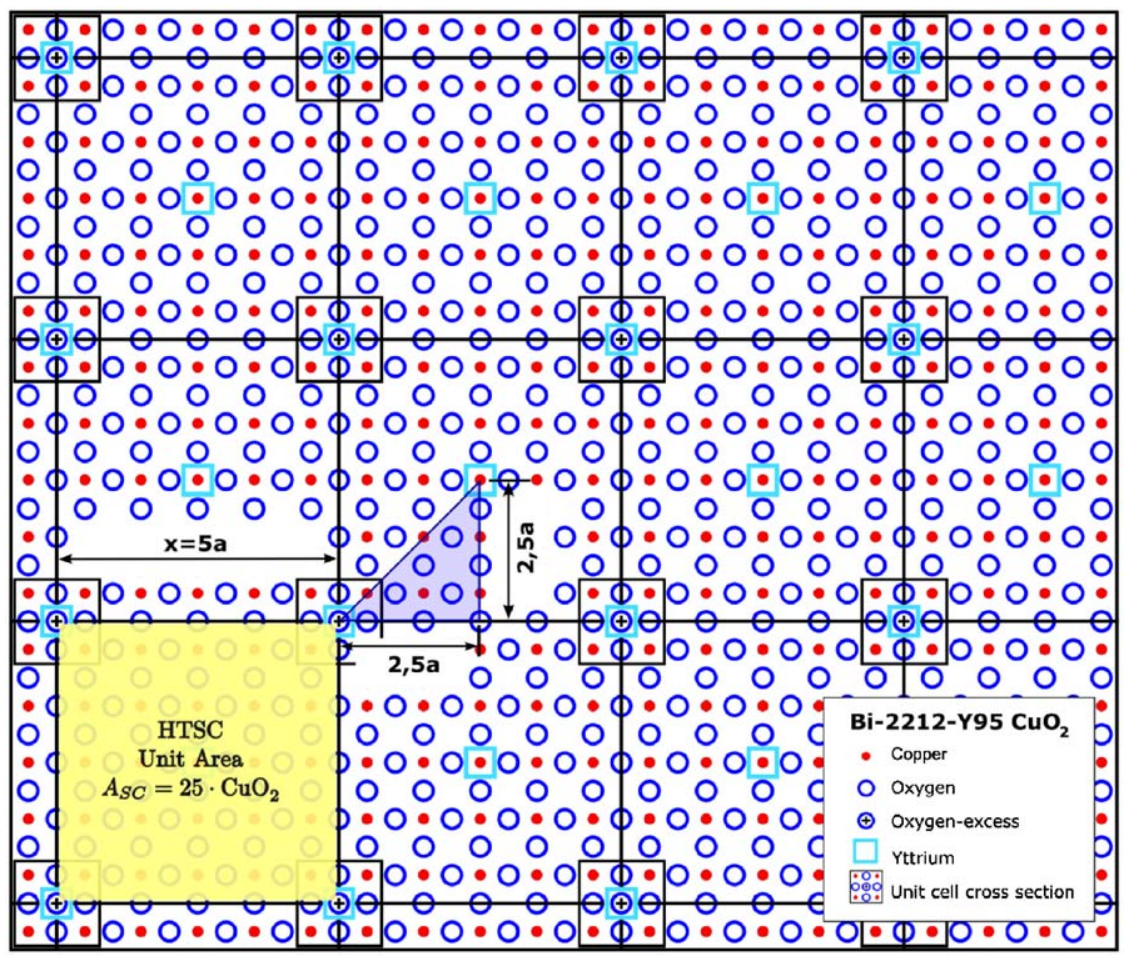

Fig. 4. Superconducting $\mathrm{CuO}_{2}$ plane of $\mathrm{Bi}-2212-\mathrm{Y} 95(a \approx b=0.541 \mathrm{~nm})$. The distance between oxygen excess atoms is the superconducting resonance length $x=2.70 \mathrm{~nm}$. The superconducting unit area has the size of $25 \cdot \mathrm{CuO}_{2}$ unit areas.

indicates that only one $\mathrm{CuO}_{2}$ plane will be superconducting, resulting in $n=1$ for Eq. (1) as is the situation with $\mathrm{YBa}_{2} \mathrm{Cu}_{3} \mathrm{O}_{7-\delta}$ [1]. For an yttrium level of about $\Delta=0.08$, the density is $\left(\Sigma_{2}\right)^{-1}=(1-0.92 / 1.00) \equiv 8 \%$ so that $\Sigma=12.5 \approx 13=3^{2}+2^{2}=z_{3}^{2}+z_{4}^{2}$. This results in a doping distance of $x_{2}=\sqrt{ } 13 \cdot a=1.95 \mathrm{~nm}$. The Moiré requirement according to Eq. (2) results in $z_{5}=z_{6}=z_{7}=1$ and $z_{8}=0$ illustrated in Fig. 3 with $x_{3}=x_{1}=2.76 \mathrm{~nm}$ and a HTSC unit area of $A_{\mathrm{SC}}=26 \cdot \mathrm{CuO}_{2}$. The calculated transition temperature $T_{c}($ calc. $)=91.2 \mathrm{~K}$ compares well with the experimental derived values.

\section{2. $B i-2212-Y 95$}

For this material, the oxygen doping level is only slightly higher, with a value of $\delta=0.16-0.17 \approx 16.5$ resulting in $\left(\Sigma_{1}\right)^{-1} \equiv 4 \%$, so that $\Sigma_{1}=25=5^{2}+0^{2}$ and the doping distance results in $x_{1}=\sqrt{ } 25 \cdot a=2.70 \mathrm{~nm}$. The Y-atom content has been determined very accurately to $8 \%$ which yields an yttrium distribution as illustrated in Fig. 4 and a HTSC unit area of $A_{\mathrm{SC}}=25 \cdot \mathrm{CuO}_{2}$.

\section{Discussion}

The calculated transition temperatures compare well with the experimental data and match Eq. (1) results and fit on the correlation curve in [2]. This result supports the suggestion that the superconducting $\mathrm{CuO}_{2}$ highway contains superconducting current channels where carriers are moving parallel and in phase at the same velocity. Further analysis could determine if Eq. (1) could be derived from the density of states in a 1D quantum wire [17].

Pure $\mathrm{Bi}_{2} \mathrm{Sr}_{2} \mathrm{CaCu}_{2} \mathrm{O}_{8+\delta}(\mathrm{Bi}-2212)$ has a transition temperature of $T_{c}=82.5 \mathrm{~K}$, but if it is doped with Y-atoms it reaches $T_{c}=91-95 \mathrm{~K}$. This $T_{c}$ value is very similar to $\mathrm{YBa}_{2} \mathrm{Cu}_{3} \mathrm{O}_{7-\delta}$ with $T_{c}=93 \mathrm{~K}$ [1]. Similarly, La-doped $\mathrm{Bi}_{2} \mathrm{Sr}_{1.6} \mathrm{La}_{0.4} \mathrm{CuO}_{6+\delta}$ has a $T_{c}=36.5 \mathrm{~K}$ and $\mathrm{La}_{1.85} \mathrm{Sr}_{0.15} \mathrm{CuO}_{4}$ (LSCO) displays $T_{c}=35-40 \mathrm{~K}$. Obviously the dopants yttrium and lanthanum determine the maximum transition temperature by increasing 
(Y-atom) or decreasing (La-atom) the maximum carrier velocity as suggested in [1-4].

\section{References}

[1] H.P. Roeser, F. Hetfleisch, F.M. Huber, M.F. von Schoenermark, M. Stepper, A. Moritz, A.S. Nikoghosyan, Acta Astronautica 62/12 (2008) 733-736.

[2] H.P. Roeser, F. Hetfleisch, F.M. Huber, M.F. von Schoenermark, M. Stepper, A. Moritz, A.S. Nikoghosyan, Correlation between oxygen excess density and critical transition temperature in superconducting $\mathrm{Bi}-2201, \mathrm{Bi}-2212$ and $\mathrm{Bi}-2223$, Acta Astronautica 63 (2008) 1372-1375.

[3] H.P. Roeser, F.M. Huber, M.F. von Schoenermark, A.S Nikoghosyan, M. Toberman, Fluorine-doped structure in ironbased high temperature superconductors, Acta Astronautica 64 (2009) 391-394.

[4] H.P. Roeser, P. Leschinski, F.M. Huber, M.F. von Schoenermark, A.S. Nikoghosyan, M. Toberman, Oxygen deficiency structure in iron-based high temperature superconductor $\mathrm{GdFeAsO}_{1-\delta}$, Acta Astronautica (2008), doi:10.1016/j.actaastro.2008.11.010.

[5] H. Eisaki, N. Kaneko, D.L. Feng, A. Damascelli, P.K. Mang, K.M. Shen, Z.X. Shen, M. Greven, Effect of chemical inhomogeneity in bismuth-based copper oxide superconductors, Physical Review B 69 (2004) 064512.

[6] Y. Ando, T. Murayama, Nonuniversal power law of the Hall scattering rate in a single-layer cuprate $\mathrm{Bi}_{2} \mathrm{Sr}_{2-x} \mathrm{La}_{x} \mathrm{CuO}_{6}$, Physical Review B 60 (10) (1999) R6991.

[7] S. Ono, Y. Ando, T. Murayama, F.F. Balakirev, J.B. Betts, G.S. Boebinger, Physical Review Letters 85 (3) (2000) 638-641.

[8] Y. Arao, M. Tange, M. Yokoshima, H. Ikeda, R. Yoshizaki, Optimization of the $\mathrm{Bi}-2201$ superconductors with $\mathrm{Pb}$ and $\mathrm{La}$ co-doping, Physica C 426-431 (2005) 351-354.
[9] F. Jean, G. Collin, M. Andrieux, N. Blanchard, J.F. Marucco, Physica E 339 (2000) 269-280.

[10] H. He, Y. Sidis, P. Bourges, G.D. Gu, A. Ivanov, N. Koshizuka, B. Liang, C.T. Lin, L.P. Regnault, E. Schoenherr, B. Keimer, Resonant spin excitation in an overdoped high temperature superconductor, Physical Review Letters 86 (8) (2001) 1610-1613.

[11] P.D. Johnson, T. Valla, A.V. Fedorov, Z. Yusof, B.O. Wells, Q. Li, A.R. Moodenbaugh, G.D. Gu, N. Koshizuka, C. Kendziora, S. Jian, D.G. Hinks, Physical Review Letters 87 (17) (2001) 177007.

[12] A.D. Gromko, A.V. Fedorov, Y.D. Chuang, J.D. Koralek, Y. Aiura, Y. Yamaguchi, K. Oka, Y. Ando, D.S. Dessau, Physical Review B 68 (2003) 174520.

[13] H.F. Fong, P. Bourges, Y. Sidis, L.P. Regnault, A. Ivanov, G.D Gull, N. Koshizuka, B. Keimer, Nature 398 (1999) 588-591.

[14] J. Hwang, T. Timusk, G.D. Gu, High-transition-temperature superconductivity in the absence of magnetic-resonance mode, Nature 427 (2004) 714-717.

[15] N. Miyakawa, J.F. Zasadzinski, L. Ozyuzer, P. Guptasarma, D.G. Hinks, C. Kendziora, K.E. Gray, Predominantly superconducting origin of large energy gaps in underdoped $\mathrm{Bi}_{2} \mathrm{Sr}_{2} \mathrm{CaCu}_{2} \mathrm{O}_{8+\delta}$ from tunnelling spectroscopy, Physical Review Letters 83 (5) (1999) 1018-1021.

[16] A. Lanzara, P.V. Bogdanov, X.J. Zhou, S.A. Kellar, D.L. Feng, E.D. Lu, T. Yoshida, H. Eisaki, A. Fujimori, K. Kishio, J.I Shimoyama, T. Noda, S. Uchida, Z. Hussain, Z.X. Shen, Evidence for ubiquitous strong electron-phonon coupling in high-temperature superconductors, Nature 412 (2001) 510-514.

[17] H.P. Roeser, F.M. Huber, M.F. von Schoenermark, A.S Nikoghosyan, Density of states in a 1D high temperature superconducting quantum wire, Acta Astronotica (2008), to be submitted for publication. 